\title{
Modelo predictivo del espesor de la capa de óxido y microdureza en aluminio Al3003-B14 y Al6063-T6 anodizado usando análisis multifactorial
}

\author{
Oxide film thickness and microhardness prediction model of Al3003-B14 and \\ Al6063-T6 anodized aluminum using multifactorial analysis \\ Leonardo Eladio Vergara Guillén $^{1} \quad$ Luis Manuel Nerey Carvajal ${ }^{1}$ \\ Víctor Manuel Guedez Torcates ${ }^{2}$ \\ Recibido 20 de abril de 2010, aceptado 8 de abril de 2011 \\ Received: April 20, $2010 \quad$ Accepted: April 8, 2011
}

\begin{abstract}
RESUMEN
En esta investigación se modela a partir de los parámetros del proceso el espesor de la capa de óxido y la microdureza de los aluminios Al3003 y Al6063 anodizados. Para ello se realizaron estudios de la microdureza y espesor de capa de la superficie anodizada, utilizando técnicas de análisis multifactorial y diseño robusto. Se establecieron los siguientes niveles de los parámetros del proceso: temperatura $\left[15^{\circ} \mathrm{C}\right.$, $25^{\circ} \mathrm{C}$ ], tiempo [30 min; $60 \mathrm{~min}$ ], concentración de electrolito [1,2 M; $2 \mathrm{M}$ ], densidad de corriente [1 Amp/ $\mathrm{dm}^{2} ; 3 \mathrm{Amp} / \mathrm{dm}^{2}$ ], aluminio [Al3003, Al6063] y como variable de ruido, la deformación plástica [0\%, $10 \%, 20 \%, 30 \%$ ]. Se propuso un diseño fraccionado $2^{7-2}$ mixto, con el cual se realizaron un total de 48 pruebas usando soluciones electrolíticas de ácido sulfúrico. La medición de microdureza se realizó con un indentador Vickers con carga de $400 \mathrm{~g}$; el espesor de la capa de óxido se captó mediante microscopia electrónica. A los resultados se les realizó un análisis de varianza (ANOVA), para determinar los factores significativos y la robustez de los efectos. Se encontraron resultados de microdureza [HV] [85,74-308,87]; y espesor de óxido $[\mu \mathrm{m}][12,82-94,69]$. Finalmente se muestran los modelos de predicción de cada una de las respuestas en función de los factores significativos; estas ecuaciones permitirán seleccionar la microdureza y espesor de la capa de óxido para cumplir los requerimientos de un producto particular mediante una selección apropiada de los parámetros del proceso.
\end{abstract}

Palabras clave: Aluminio, anodizado, diseño factorial, ANOVA, robustez.

\section{ABSTRACT}

In this research, the thickness of the oxide layer and the microhardness of anodized aluminum Al3003 and Al6063 are modeled based on process parameters. To this end, studies of the microhardness and the thickness layer of the anodized surface were made, via techniques of multifactorial analysis and robust design. The following levels of the process parameters were established: temperature $\left[15^{\circ} \mathrm{C}, 25^{\circ} \mathrm{C}\right]$, time [30min; 60min], electrolyte concentration [1,2M;2M], current density [1 Amp/dm $\left.{ }^{2} ; 3 \mathrm{Amp} / \mathrm{dm}^{2}\right]$, aluminum [Al3003, Al6063], and as a noise parameter, the plastic deformation [0\%, 10\%, 20\%, 30\%]. A combined fractional design $2^{7-2}$ was proposed, based on which a total of 48 tests were performed using sulfuric acid electrolytic solutions. The measurement of microhardness was performed using a Vickers indenter loaded at 400g, and the thickness of the oxide layer was captured using electron microscopy. Variance analysis (ANOVA) was applied to the results in order to determine the significant factors and the robustness of the effects. Results of microhardness [HV] [85,74-308,87]; and the oxide layer thickness $[\mu m][12,82-94,69]$ were determined. Finally, the equations for the prediction models are shown for each response as a function of the significant factors, these equations will allow the selection of the

1 Escuela de Diseño Industrial. Universidad de los Andes. Código postal: 5101. Teléfono: +582742401902 . Correo electrónico: lvergara@ula.ve; nerey@cptm.ula.ve

2 Escuela de Ingeniería Mecánica. Universidad de los Andes. Código postal: 5101. Teléfono: +582742402936. Correo electrónico: guedez@ula.ve 
microhardness and thickness of oxide layer to fulfill the requirements of a particular product using an appropriate selection of process parameters.

Keywords: Aluminum, anodizing, factorial design, ANOVA, robustness.

\section{INTRODUCCIÓN}

El anodizado es un proceso de oxidación por conversión, en el que la superficie de la pieza se convierte en una capa dura y porosa de óxido. Esta capa es permanente e integral con el metal base original y protege la pieza, parte o componente de aluminio, contra los agentes atmosféricos de oxidación y corrosión; genera resistencia a la abrasión y desgaste, además se puede pigmentar, y carece de toxicidad tanto en medicina como en la industria alimentaria [1-4]. El anodizado fue desarrollado para tratar el aluminio, el cual tiene características y propiedades mecánicas, físicas y químicas que lo hacen una opción de selección muy adecuada para fabricar productos con determinados requerimientos de diseño. No obstante, muchas de estas características se podrían mejorar a través de un proceso de acabado superficial de anodizado. En Venezuela, país donde se estiman 200 millones de toneladas métricas de bauxita que se pueden transformar a alúmina, materia prima del aluminio, los principales aluminios producidos son el aluminio Al3003 y el Al6063, Tabla 1. Sin embargo, en estos aluminios no se tienen referencias de combinaciones específicas en parámetros del proceso que se puedan relacionar con cambios en las características contra la corrosión o el desgaste. Igualmente, no se tienen referencias del efecto de la deformación plástica en frío en una etapa previa al proceso de anodizado y su influencia sobre la calidad del mismo. Por lo tanto, se quiere determinar cómo y en qué medida los factores del proceso (deformación plástica, material, concentración del electrolito, densidad de corriente, temperatura y tiempo) influyen en el proceso de anodizado y establecer la combinación de factores que producen los efectos más significativos sobre la microdureza y el espesor de la capa de óxido.

La importancia de este estudio radica en aumentar el rango de aplicación de los aluminios Al3003 y Al6063 de acuerdo con los requisitos funcionales particulares del producto final. En principio, estableciendo los valores requeridos de microdureza y espesor de la capa de óxido en la superficie del
Tabla 1. Composición de los aluminios estudiados.

\begin{tabular}{|c|c|c|}
\hline $\begin{array}{c}\text { Composición\ } \\
\text { Aluminio }\end{array}$ & $\begin{array}{c}\text { Al3003 } \\
\text { (ALCASA) }\end{array}$ & Al6063 \\
\hline $\mathrm{Si}$ & 0,20 & 0,40 \\
\hline $\mathrm{Fe}$ & 0,65 & 0,35 \\
\hline $\mathrm{Cu}$ & 0,128 & 0,10 \\
\hline $\mathrm{Mn}$ & 1,042 & 0,10 \\
\hline $\mathrm{Mg}$ & 0,003 & 0,68 \\
\hline $\mathrm{Cr}$ & 0,0005 & 0,10 \\
\hline $\mathrm{Zn}$ & 0,008 & 0,10 \\
\hline $\mathrm{Ti}$ & 0,013 & 0,10 \\
\hline Otro & 0,15 & 0,15 \\
\hline Tratamiento & $\mathrm{H}-14$ & $\mathrm{~T}-6$ \\
\hline
\end{tabular}

H-14: Endurecido por deformación

T-6: Maduración artificial: Estado T6, 8 horas a $170{ }^{\circ} \mathrm{C} \pm 5{ }^{\circ} \mathrm{C}$ o 6 horas a $185{ }^{\circ} \mathrm{C} \pm 5^{\circ} \mathrm{C}$.

material (los cuales generalmente son asociados con resistencia a desgaste y a la corrosión que el material inicialmente no posee). Posteriormente, para llegar a estos resultados será necesario aplicar en la etapa final de manufactura un proceso de anodizado, seleccionando la combinación de parámetros (tipo de aluminio, densidad de corriente, concentración del electrolito, temperatura, tiempo) que producen los resultados deseados y satisfacen los requisitos funcionales de la pieza, parte o componente. Para determinar esta combinación de parámetros se modela la respuesta de microdureza y capa de óxido usando técnicas de análisis multifactorial.

\section{Importancia del espesor de la capa de óxido y de la microdureza}

El aluminio y sus aleaciones quedan protegidos naturalmente por una capa de óxido, cuando son expuestos al aire. Sin embargo, esta capa de óxido natural es delgada y heterogénea y no ofrece protección suficiente contra ambientes agresivos [5]. Es por ello que mediante el proceso electroquímico de anodizado se puede convertir un espesor de óxido mayor y más homogéneo, el cual puede mejorar 
algunas características como son la resistencia a la corrosión, al desgaste y la facilidad de limpieza superficial del aluminio. Asimismo, permite crear capas dieléctricas y capas aislantes tanto térmicas como eléctricas en la industria electrónica [6-7]. Sin embargo, la reflectividad se reduce debido al incremento del espesor [8-9].

Igualmente, la dureza de la capa de óxido es una propiedad muy importante porque indica de forma cualitativa su resistencia mecánica, es una medida de la resistencia a la deformación permanente, de su resistencia al rayado y, además, es un índice de la resistencia al desgaste abrasivo [10-13]. Diferentes estudios muestran que generalmente un aumento de la dureza, también aumenta la resistencia al desgaste, abrasión y vida útil de un material [14-16].

\section{Importancia del análisis multifactorial}

Tradicionalmente, la estrategia para hacer este tipo de experimentos es el enfoque de un factor a la vez. Este método consiste en seleccionar un punto de partida, o línea base de los niveles, para cada factor, para después variar sucesivamente cada factor en su rango, manteniendo constantes los factores restantes en el nivel base. Después de haber realizado todas las pruebas, se construye por lo general una serie de gráficas en las que se muestra la forma en que la variable de respuesta es afectada al variar cada factor manteniendo los demás factores constantes. La principal desventaja de la estrategia de un factor a la vez es que no se puede tomar en consideración cualquier posible interacción de factores. Hay una interacción cuando uno de los factores no produce el mismo efecto en la respuesta con niveles diferentes de otro factor. Por lo tanto, cuando en los experimentos se estudian los efectos de más de dos factores (seis para este caso en particular), los diseños factoriales son más eficientes. El diseño factorial de experimentos se usa con el objetivo de aumentar significativamente la calidad y la productividad simultáneamente, ya que se basa en el manejo de las variables que permiten mejorar la calidad, al mínimo coste. Es una herramienta ampliamente utilizada en la actualidad, y es considerada una pieza clave en la ingeniería de calidad. Aporta una metodología para reducir la variabilidad propia de las características de calidad de los productos, y las que originan los procesos sobre los productos [17].
Dentro del diseño factorial se utilizará el enfoque del diseño robusto [17-18], el cual se usa para resolver problemas en los que existe clasificación de las variables que influyen en un proceso o producto como variables de control (factores controlables) y de ruido (factores no controlables) para después encontrar los ajustes de variables controlables que minimizan la variabilidad transmitida a la respuesta por las variables no controlables. Se establece el supuesto de que las variables de ruido que no son controlables en el sistema a gran escala pueden controlarse para fines de experimento. El diseño robusto se ha aplicado para estudiar procesos de manufactura en los que los parámetros o factores pueden ser independientes o interdependientes como por ejemplo para optimizar los parámetros de procesos de fundición, soldadura por puntos y laminado y doblado [19-22].

Asimismo, se ha demostrado dentro de la metodología del diseño factorial que ciertas interacciones de orden superior son insignificantes, por lo que se puede obtener información de las interacciones principales y de orden inferior corriendo únicamente una fracción del experimento factorial completo $[18,23]$. Estos diseños factoriales fraccionados se encuentran entre los tipos de diseños de uso más generalizado en el diseño de productos y procesos y en el mejoramiento de procesos $[4,5,24]$.

\section{PARTE EXPERIMENTAL}

Se establecieron los siguientes parámetros y sus niveles como los más importantes del proceso considerando la experiencia de especialistas de la industria [18,23]: el aluminio C:[Al3003, Al6063], la concentración del electrolito D:[1,2 M; $2 \mathrm{M}]$, la densidad de corriente E:[1 Amp/dm $\left.{ }^{2} ; 3 \mathrm{Amp} / \mathrm{dm}^{2}\right]$, la temperatura $\mathrm{F}:\left[15^{\circ} \mathrm{C}, 25^{\circ} \mathrm{C}\right]$, el tiempo G:[30 min; $60 \mathrm{~min}]$, y como variable de ruido, la deformación plástica X:[0\%, 10\%, 20\%, 30\%].

Se seleccionó un diseño factorial fraccionado $2^{7-2}=32$ pruebas, lo cual significa que se realizará sólo un cuarto de las pruebas que requeriría un experimento tradicional (128 experimentos), más cuatro puntos centrales a los factores cuantitativos en cada aluminio para poder observar la posible pérdida de linealidad en los efectos de los factores. Posteriormente se hacen ocho réplicas, para la 
validación de las respuestas, lo que permite una estimación del error. Los 48 experimentos se realizaron de manera aleatoria.

Por lo tanto, se utilizaron 48 probetas con dimensiones iniciales de $(25 \mathrm{~mm}) \times(15 \mathrm{~mm}) \times(1,56 \mathrm{~mm})$, para que su calentamiento no influya en la temperatura del electrolito y para observarlas en el microscopio de barrido electrónico MBE. De cada aluminio se tomaron cuatro grupos de seis (6) probetas con los porcentajes de reducción especificados. El área matemática de cada probeta es aproximadamente de $500 \mathrm{~mm}^{2}=0,05 \mathrm{dm}^{2}$, debido a que una cara se cubrió con resina epóxica. Las probetas se limpian con alcohol absoluto por inmersión, se decapan por 30 s en hidróxido de sodio $(\mathrm{NaOH})$ al $10 \%$ peso, se lavan con agua destilada, se aclaran con ácido nítrico $15 \%$ por $15 \mathrm{~s}$, se lavan con agua destilada, se coloca en la cuba electrolítica, se anodiza según los niveles de los factores indicados para la prueba, se lava con agua, se seca al aire y se almacena y codifica para su posterior análisis.

Para la medición de la microdureza en cada probeta se utilizó el ensayo de microdureza Vickers, y se tomó el promedio de cinco (5) mediciones para caracterizar cada una. La media de la microdureza antes del anodizado fue $48 \mathrm{HV}$ y $102,64 \mathrm{HV}$ para el A13003 y A16063, respectivamente.

Para la medición del espesor se hace un examen microscópico basado en la norma ASTM B487-85, la cual consiste en cortar una porción de la probeta de prueba y prepararla mediante pulido para luego medirla con el microscopio MBE. Las observaciones por MBE se realizaron en un equipo Hitachi S-2500 con electrones secundarios y el análisis químico utilizando un equipo Thermo-Noran acoplado al MBE. La medida tomada de espesor en cada probeta es el promedio de cinco (5) mediciones realizadas. El procedimiento se observa en la Figura 1.

\section{Análisis de varianza (ANOVA): Procedimiento para determinar los efectos que produce cada factor y su significancia}

Las ecuaciones para determinar el efecto $(E f)$ que produce cada factor sobre la respuesta se muestran a continuación:

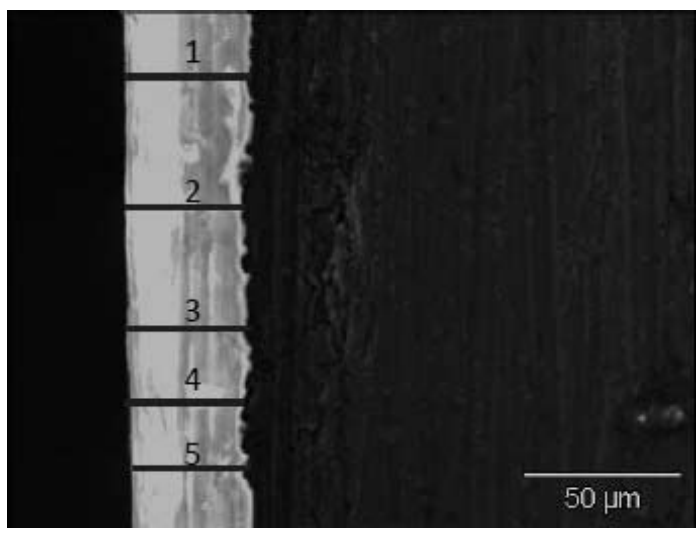

Figura 1. Determinación del espesor. Magnificación: 1500X.

Para los factores cualitativos:

$$
E f_{c}=\sum_{i=1}^{n=48} \frac{E_{i} \cdot \text { Factor } C_{i}}{\frac{n}{2}}
$$

Para los factores cuantitativos:

$$
E f_{d} \sum_{i=1}^{n=48} \frac{E_{i} \cdot \text { Factor } D_{i}}{16}
$$

La suma total de los cuadrados es:

$$
S S_{t}=\sum_{i=1}^{n=48} E_{i}^{2}-\frac{Y^{2}}{48}
$$

La ecuación que define la suma de cuadrados para cada término cualitativo, utilizando como ejemplo el factor C, será:

$$
S S_{c}=\frac{\left(\sum_{i=1}^{n=48} E_{i} \cdot \text { Factor } C_{i}\right)^{2}}{48}
$$

Y para los factores cuantitativos, utilizando como ejemplo el factor D, será:

$$
S S_{D}=\frac{\left(\sum_{i=1}^{n=48} E_{i} \cdot \text { Factor } D_{i}\right)^{2}}{32}
$$

La suma de los cuadrados para el modelo es la suma de los cuadrados de todos los factores y la suma de los cuadrados para el error es el total menos el modelo:

$$
S S_{\text {modelo }}=\sum S S_{\text {factores }}
$$




$$
S S_{\text {Error }}=S S_{t}=S S_{\text {modelo }}
$$

La media de los cuadrados para cada elemento, por ejemplo para el modelo:

$$
M S_{\text {modelo }}=\frac{S S_{\text {modelo }}}{V_{\text {modelo }}}=\frac{S S_{\text {modelo }}}{32}
$$

Donde V son los grados de libertad

Y la media de los cuadrados para el error será:

$$
M S_{\text {error }}=\frac{S S_{\text {error }}}{V_{\text {error }}}=\frac{S S_{\text {error }}}{7}
$$

El estadístico de prueba es $\mathrm{F}_{0}$, definido como:

$$
F_{\text {factor }}=\frac{M S_{\text {factor }}}{M S_{\text {error }}}
$$

El estadístico de prueba $\mathrm{F}_{0}$ se utiliza para hacer una prueba de significación o de hipótesis. En esta prueba se puede determinar la probabilidad $\mathrm{P}$ mediante tablas estadísticas de una distribución $\mathrm{F}$, con los grados de libertad del error $\mathrm{V}_{\text {error }} \mathrm{y}$ del factor $\mathrm{V}_{\text {factor }} ; \mathrm{P}\left(\mathrm{F}_{0}\right.$, $\mathrm{V}_{\text {factor }}, \mathrm{V}_{\text {error }}$ ) indica la probabilidad de que el factor produzca el efecto calculado en la respuesta cuando se tiene una hipótesis nula $\mathrm{H}_{0}: \mu_{1}=\mu_{2}$ verdadera, es decir, cuando los cambios de nivel en el factor en realidad no afectan la respuesta promedio (cuando el factor no es significativo). Este valor P se compara con un nivel de significancia específico como, por ejemplo, un $\alpha=0.1$ o $\alpha=0.05$. Si P $<\alpha$ se considera el factor significativo (en realidad un cambio de nivel en el factor afecta la respuesta promedio) y se rechaza la hipótesis $\mathrm{H}_{0}$.

\section{EFECTOS SOBRE LA MICRODUREZA}

En el estudio de la microdureza del anodizado los mayores valores se encontraron en A16063, el cual es de naturaleza más dura que el Al3003, casi dos veces más duro antes del anodizado. Los efectos promedio de la variación de nivel de cada factor sobre la microdureza de la capa formada se pueden observar en la Figura 2.

Los factores más significativos de acuerdo al análisis de varianza (ANOVA) Tabla 2 fueron los siguientes: (a) la densidad de corriente en el nivel de $3 \mathrm{~A} / \mathrm{dm}^{2}$, aumenta la microdureza debido a que la intensidad de corriente incrementa la energía necesaria para

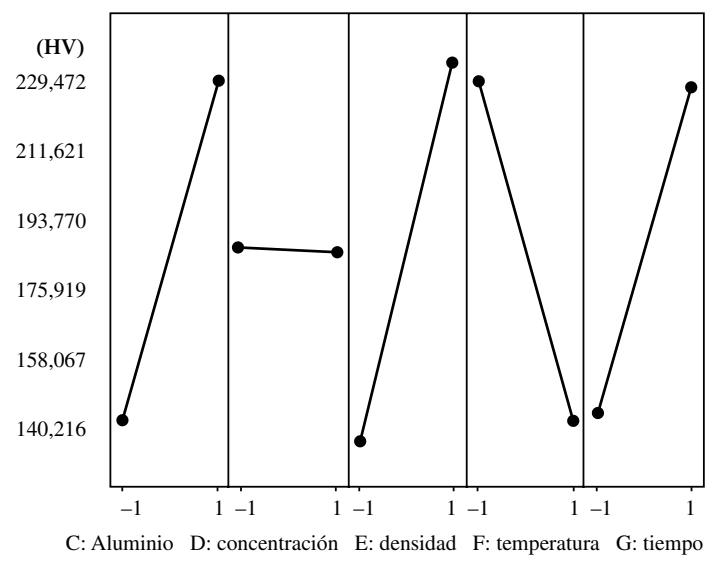

Figura 2. Respuesta promedio de microdureza según el nivel de los factores (-1: Nivel Bajo;1: Nivel Alto).

el movimiento de los iones que forman la capa de alúmina, la cual se endurece a medida que crece; (b) la temperatura en su nivel menor de $15^{\circ} \mathrm{C}$ aumenta la microdureza porque no se reduce la formación de alúmina, esto es debido a que es menor el efecto de disolución producido por el ácido sulfúrico ya que es menos activa la reacción heterogénea de oxidoreducción sobre la superficie de la pieza; (c) un tiempo de 60 minutos aumenta la microdureza ya que la alúmina sigue creciendo dentro el rango estudiado; (d) En la Figura 3 se muestra que la respuesta de microdureza en el aluminio 3003 no es sensible al factor deformación, es decir, que si se considera la deformación inicial como un ruido, la respuesta de microdureza se puede predecir en un objeto anodizado, independientemente de la variabilidad de la deformación en el mismo. Sin embargo, en aluminio 6063 la microdureza sí es sensible al factor deformación, y esta aumenta en función directa con la deformación, por lo que la respuesta de microdureza

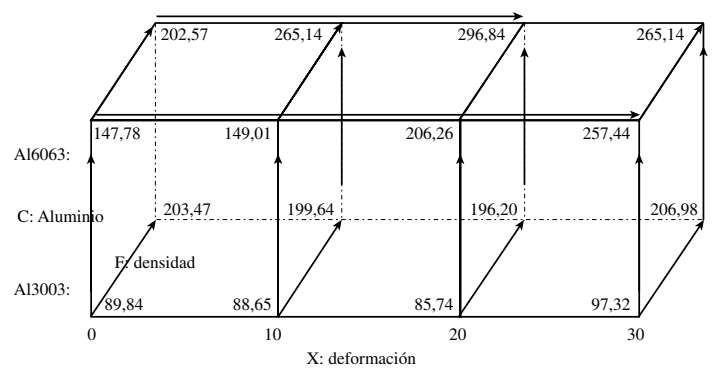

Figura 3. Gráfico cúbico de las variables de interacción deformación vs. densidad de corriente y tipo aluminio. 
no será uniforme en un elemento que presente variabilidad o no uniformidad en la deformación antes del anodizado cuando se utilice aluminio 6063. En este aluminio aumenta el valor de la dureza debido a la deformación previa porque tiene un coeficiente de resistencia y de endurecimiento por deformación mayor que el aluminio 3003.

Tabla 2. Información de los términos significativos en microdureza según ANOVA.

\begin{tabular}{|l|c|c|c|}
\hline Término & Efecto & $\mathbf{F}_{\mathbf{0}}$ & Valor P \\
\hline X:deformación & 62,16 & 4,07 & 0,05 \\
\hline C:Aluminio & 78,79 & 43,10 & 0,0003 \\
\hline E:Densidad & 89,25 & 44,24 & 0,0003 \\
\hline F:temperatura & $-78,90$ & 34,57 & 0,0006 \\
\hline G:tiempo & 76,85 & 32,80 & 0,0007 \\
\hline XC & 35,58 & 2,62 & 0,14 \\
\hline
\end{tabular}

A continuación se seleccionan los valores de los coeficientes de regresión que resultaron ser significativos de los calculados en la Tabla 2 para el estudio de la microdureza. Estos se utilizan para definir una ecuación que permita modelar la respuesta de microdureza en función de los términos significativos individuales o de interacción. La ecuación resultante que modela la respuesta de microdureza es:

$Y$ microdureza $(H V)=181,54+11,0087 x_{a}+$ $22,4654 x_{b}+4,3296 x_{a} x_{b}+39,8575 x_{c}+44,6278 x_{e}$ $-39,4534 x_{f}+41,1578 x_{g}+3,5675 x_{a c}+13,7958 x_{b c}$ $-10,9641 x_{c e}+10,2047 x_{c f}-10,0384 x_{c g}$

donde $x_{a}, x_{b}, x_{c} \ldots x_{c g}$, son variables codificadas (-1 $\left.\leq x_{i} \leq+1\right)$, que representan a los términos $\mathrm{A}, \mathrm{B}, \mathrm{C} \ldots$. CG. Y la deformación equivalente se determina según la Tabla 3.

Tabla 3. El factor deformación $\mathrm{X}$ con cuatro niveles expresado como dos factores de dos niveles.

\begin{tabular}{|c|c|c|c|}
\hline Factor A & Factor B & $\mathbf{A B}$ & Factor $\mathbf{X}$ \\
\hline-1 & -1 & +1 & 0 \\
\hline+1 & -1 & -1 & 10 \\
\hline-1 & +1 & -1 & 20 \\
\hline+1 & +1 & +1 & 30 \\
\hline
\end{tabular}

Para el cálculo de la correlación o proporción de la variabilidad explicada por el modelo se utiliza el parámetro $R^{2}$

$$
R^{2}=\frac{2,95 E 5}{3,16 E 5}=0,93
$$

El estadístico de $R^{2}$ está ajustado para los factores significativos del modelo de regresión e indica que la ecuación predice el comportamiento de la microdureza en función de los factores significativos con un valor de $R^{2}=0,93$.

Los límites de microdureza encontrados varían entre 83,92-308,87 HV para el Al3003 y entre 102,64308,87 HV para el Al6063.

En la Tabla 4 se observan los porcentajes de concentración atómica de la capa anodizada en dos aluminios analizados; se puede observar que los mayores porcentajes corresponden a oxígeno y aluminio, ambos componentes principales de la alúmina, y concentraciones considerables de azufre que no estaban en el material base.

Tabla 4. Análisis químico en porcentaje de concentración atómica de la capa anodizada en aluminios estudiados.

\begin{tabular}{|c|c|c|c|c|c|c|c|}
\hline Aleación & $\mathbf{O}$ & $\mathbf{A l}$ & $\mathbf{S i}$ & $\mathbf{S}$ & $\mathbf{M n}$ & $\mathbf{M g}$ & Otro \\
\hline 3003 & 56,4 & 37,3 & 0,20 & 5,10 & 0,35 & 0 & 0,65 \\
\hline 6063 & 58,6 & 35,4 & 0,40 & 4,57 & 0 & 0,68 & 0,35 \\
\hline
\end{tabular}

\section{EFECTO SOBRE EL ESPESOR DE LA CAPA DE ÓXIDO}

En la Tabla 5 se muestran los factores más significativos en el espesor de la capa de óxido, los cuales son: (a) la concentración de la solución $2 \mathrm{M}$ aumenta el espesor cuando se mantienen constantes los demás parámetros del proceso, (b) la densidad de corriente en $3 \mathrm{~A} / \mathrm{dm}^{2}$ produce espesores altos, (c) la temperatura en $15{ }^{\circ} \mathrm{C}$ aumenta el espesor, (d) cuando se trabaja con un tiempo en el nivel de 60 minutos los valores del espesor son más altos y (e) el espesor promedio es ligeramente mayor cuando se trabaja con A16063. 
Tabla 5. Información de los términos significativos en espesor según ANOVA.

\begin{tabular}{|l|r|r|r|}
\hline Término & Efecto & \multicolumn{1}{c|}{$\mathbf{F}_{\mathbf{0}}$} & Valor $\mathbf{P}$ \\
\hline C:Aluminio & 7,67 & 52,31 & 0,0002 \\
\hline D:Concentracion & 14,31 & 189,16 & $2,53 \mathrm{E}-06$ \\
\hline E:Densidad & 27,22 & 4,74 & 0,066 \\
\hline F:temperatura & $-4,31$ & 90,47 & $2,97 \mathrm{E}-05$ \\
\hline G:tiempo & 18,82 & 18,81 & 0,0034 \\
\hline CE & 4,24 & 4,60 & 0,0691 \\
\hline CF & 6,49 & 10,77 & 0,0134 \\
\hline DE & 13,15 & 44,19 & 0,0003 \\
\hline DF & $-10,49$ & 28,09 & 0,0011 \\
\hline DG & 5,76 & 8,48 & 0,0226 \\
\hline CDE & $-4,40$ & 4,95 & 0,0615 \\
\hline CDG & $-10,79$ & 29,744 & 0,001 \\
\hline
\end{tabular}

La Figura 4 muestra que cuando la densidad es baja, el aumento de nivel de concentración no afecta la dimensión del espesor. Sin embargo, cuando la densidad de corriente está en el nivel alto, el aumento del espesor es sustancial. Esto se debe a que la densidad de corriente da la energía necesaria para el movimiento de iones que forman la capa de óxido, a baja concentración cuando hay pocos iones que mover el aumento de densidad de corriente sólo puede crear capas anodizadas pequeñas; a medida que aumenta la concentración se observa que los espesores se hacen mayores debido a que la energía está siendo utilizada para mover más iones. Esto es más notable cuando la concentración está en $2 \mathrm{M}$ donde el nivel alto de densidad de corriente en $3 \frac{A}{d m^{2}}$ puede producir los mayores espesores promedios.

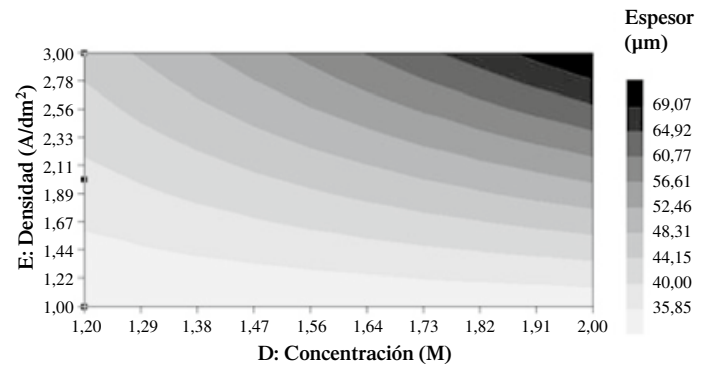

Figura 4. Diagrama de contorno entre los factores concentración del electrolito y la densidad de corriente.
En base a los factores que resultaron significativos, se seleccionan los coeficientes de regresión calculados, los cuales se utilizan para definir una ecuación que permita modelar la respuesta del espesor de la capa de óxido en función de los términos significativos individuales o de interacción.

La ecuación resultante que modela la respuesta del espesor de la capa de óxido es:

$Y$ (espesor) $=38,099+3,5838 x_{c}+7,1569 x_{d}+$ $13,61 x_{e}-2,1538 x_{f}+8,765 x_{g}+2,1821 x_{a c}+0,4463 x_{b c}$ $-1,8175 x_{c d}+2,1231 x_{c e}+3,2481 x_{c f}+6,5788 x_{d e}$ $-5,245 x_{d f}+2,8825 x_{d g}+1,7262 x_{a c g}+1,7262 x_{b c g}-$ $2,2006 x_{c d e}-5,3969 x_{c d g}-11,6706 x_{c e f g}$

donde $x_{c}, x_{d} \ldots x_{c e f g}$ son variables codificadas $\left(-1 \leq x_{i} \leq+1\right)$ que representan a los términos A, B, C....CEFG. Y la deformación equivalente se determina según la Tabla 3.

Para el cálculo de la correlación o proporción de la variabilidad explicada por el modelo se usa el término $R^{2}$, el cual es función de la suma de los cuadrados de los factores significativos del modelo y la suma de los cuadrados totales.

$$
R^{2}=\frac{1,67 E 4}{1,79 E 4}=0,94
$$

El estadístico de $R^{2}$ indica que la ecuación predice el comportamiento del espesor de la capa de óxido en función de los factores significativos con un valor de $R^{2}=0,94$. Los límites del espesor de capa de óxido se encontraron entre 13,28-94,69 $\mu \mathrm{m}$ para el Al3003, tal como se muestra en las Figuras 5 y 6 .

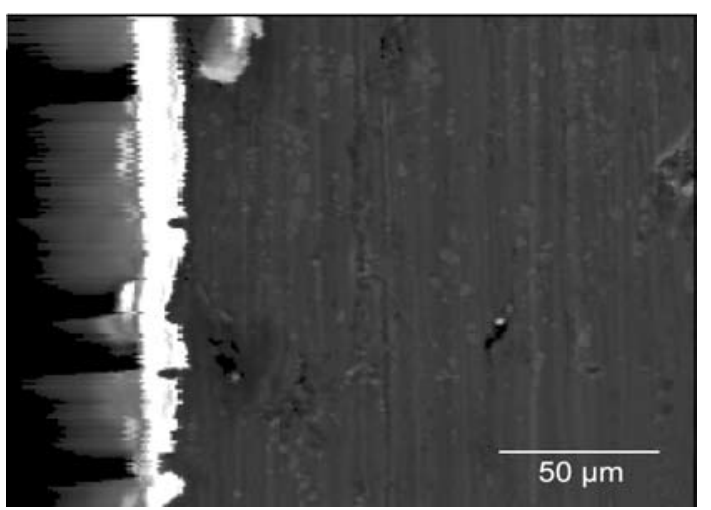

Figura 5. Espesor menor de 13,28 $\mu \mathrm{m}$ en el aluminio A13003 anodizado. Magnificación: $1500 X$. 


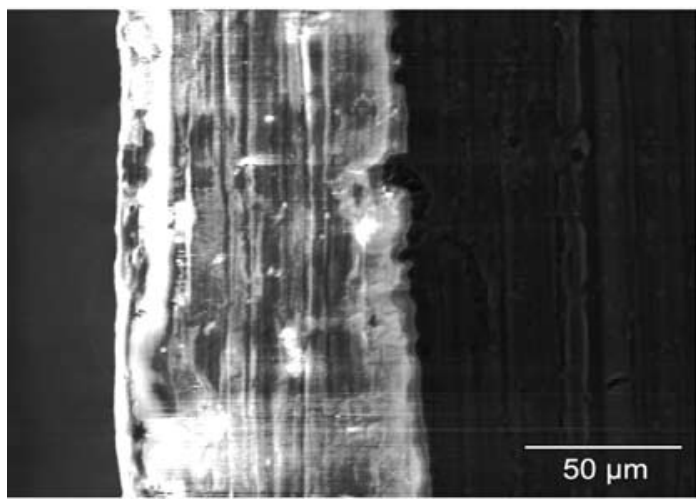

Figura 6. Espesor mayor de 94,69 $\mu$ m en el aluminio A13003 anodizado. Magnificación: $1500 \mathrm{X}$.

Asimismo, para el Al6063 se encontraron entre $12,82-76,73 \mu \mathrm{m}$, tal como se muestra en las Figuras 7 y 8.

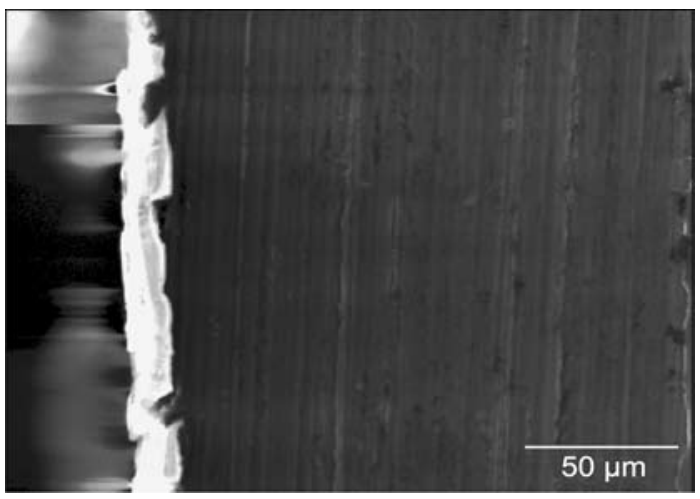

Figura 7. Espesor menor de 12,82 $\mu \mathrm{m}$ en el aluminio Al6063 anodizado. Magnificación: $1500 \mathrm{X}$
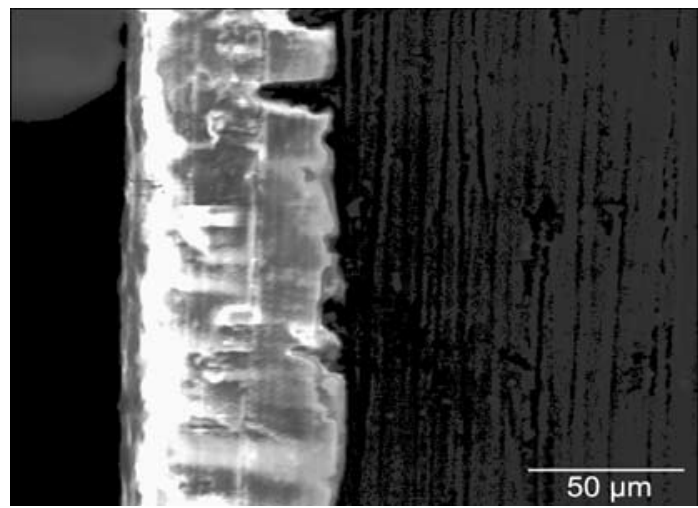

Figura 8. Espesor mayor de 76,73 $\mu \mathrm{m}$ en el aluminio Al6063 anodizado. Magnificación: $1500 \mathrm{X}$.

\section{CONCLUSIONES}

Se utilizó la metodología del diseño factorial, recurriendo al enfoque de diseño robusto, la cual es una herramienta que demostró ser de gran utilidad para la realización de esta investigación. Mediante esta metodología se determinaron los factores significativos que afectan las respuestas de microdureza y espesor de la capa de óxido anódica.

Se realizó un modelado de la microdureza, y del espesor de la capa de óxido formada en el anodizado, como una función de los parámetros del proceso que producen los efectos más significativos en cada respuesta.

Al contrastar las hipótesis planteadas se tiene que el porcentaje de deformación inicial, la cual es la variable de ruido, generó respuestas robustas en la microdureza del A13003, y espesor de la capa de óxido en ambos aluminios. Por lo tanto, tiene efecto significativo sólo sobre la microdureza del Al6063.

En el estudio de la microdureza del anodizado, los mayores valores se encontraron en A16063, el cual es de naturaleza más dura que el Al3003. Los factores más significativos resultaron: (a) la densidad de corriente cuyo nivel más alto aumenta la microdureza; (b) la temperatura cuyo valor menor aumenta la microdureza; (c) la extensión del tiempo que aumenta la microdureza. La deformación sólo fue significativa en Al6063, en el cual la reducción de área produjo endurecimiento por deformación. El aumento de la microdureza se puede utilizar para crear productos con mayor resistencia a deformación permanente; mayor resistencia al rayado, al desgaste y abrasión.

En relación al estudio del espesor de la capa de óxido, se encontraron como los factores más significativos que: (a) la concentración de la solución $2 \mathrm{M}$ aumenta el espesor cuando se mantienen constantes los demás parámetros del proceso; (b) la densidad de corriente en $3 \mathrm{~A} / \mathrm{dm}^{2}$ produce espesores altos; (c) la temperatura en $15^{\circ} \mathrm{C}$ aumenta ligeramente el espesor; (d) cuando se trabaja con un tiempo en el nivel de 60 minutos los valores del espesor son mayores, y (e) el espesor promedio es ligeramente mayor cuando se trabaja con Al6063. El hecho de 
utilizar aluminio con mayores espesores de la capa de óxido puede mejorar la resistencia a la corrosión y oxidación; la resistencia al desgaste; la facilidad de limpieza; y la capacidad de aislamiento térmico y eléctrico del material.

Asimismo, se observó que la microdureza y el espesor de la capa anodizada aumentan o disminuyen en proporción directa por los mismos factores, excepto por la concentración del electrolito que sólo es significativa para el espesor de la capa anodizada.

Finalmente, tanto para el aluminio 3003 como para el 6063 se realizaron modelos de predicción de cada una de las respuestas en función de los parámetros significativos; estas ecuaciones permitirán seleccionar la microdureza y el espesor de la capa de óxido que se formará por anodizado de acuerdo a los requerimientos del producto, por medio de la selección apropiada de los parámetros del proceso

\section{AGRADECIMIENTOS}

Al Consejo de Desarrollo Científico Humanístico y Tecnológico de la Universidad de los Andes. C.D.C.H.T-U.L.A. Mérida, Venezuela. Proyecto: $\mathrm{N}^{\circ}$ : I-933-06-02-A. y I-1194-09-02-EM.

\section{REFERENCIAS}

[1] J. Konieczny, L.A Dobrzanski, K. Labisz and J. Duszczyk. "The influence of cast method and anodizing parameters on structure and layer thickness of aluminium alloys". Journal of Materials Proccessing Technology. Vol. 157158, pp. 718-723. Diciembre, 2004.

[2] N.M. Yakovleva, L. Anicai, A.N. Yakovlev, L. Dima, E. Ya. Khanina and M. Buda. "Structural study of anodic films formed on aluminium in nitric acid electrolyte". Thin Solid films. Vol. 416, Issue 1-2, pp. 16-23. September, 2002.

[3] S. Feliú Jr., M.J. Bartolomé, J.A. González, V. López and S. Feliú. "Passivating oxide film and growing characteristics of anodic coating on aluminium alloys". Applied Surface Science. Vol. 254, Issue 9, pp. 2755-2762. February, 2008.

[4] A. Bai, C.-C. Hu, Y.-F. Yang and C.-C. Lin. "Pore diameter control of anodic aluminum oxide with ordered array of nanopores". Electrochimica Acta. Vol. 53, Issue 5, pp. 22582264. January, 2008.

[5] W. Bensalah, K. Elleuch, M. Feki, M. Wery, M.P. Gigandet and H.F. Ayedi. "Optimization of mechanical and chemical properties of sulphuric anodized aluminium using statistical experimental methods". Material Chemistry and Physics. Vol. 108, Issue 2-3, pp. 296-305. April, 2008.

[6] M. Spoelstra, E. Van Westing and J. De Wit. "Characterization of unsealed anodic oxide layers on aluminium". Materials and Corrosion. Vol. 52, Issue 9, pp. 661-666. September, 2001.

[7] M.F. Morks, A.S. Hamdy, N.F. Fahim and M.A. Shoeib. "Growth and characterization of anodic films on aluminum alloys in 5-sulfosalicylic acid solution”. Surface and Coatings Technology. Vol. 200, Issue 16-17, pp. 5071-5076. April, 2006.

[8] I. Vrublevsky, A. Jagminas, J. Schreckenbach and W.A. Gadel. "Electronic properties of electrolyte/anodic alumina junction during porous anodizing". Applied surface science. Vol. 253, Issue 10, pp. 4680-4687. March, 2007.

[9] T.S. Shih, P.-S. Wei and Y.-S. Hung. “Optical properties of anodic aluminium oxide film on AL1050 alloys". Surface and Coating Technology. Vol. 202, Issue 14, pp. 3298-3305. April, 2008.

[10] W. Smith y J. Hashemi. "Fundamentos de la ciencia e ingeniería de los materiales". McGraw Hill. México. 2004.

[11] J.F. Shackelford. "Introducción a la ciencia de materiales para ingenieros". Prentice Hall. México.1998.

[12] S. Kalpakjian y S. Scmid. "Manufactura, ingeniería y tecnología". Cuarta Edición, Pearson Educación. México. 2002.

[13] M. Groover. "Fundamentos de manufactura moderna, Materiales, procesos y sistemas". Prentice Hall. 1997.

[14] R. Mott. "Diseño de elementos de máquinas". Cuarta Edición. Pearson Educación. México. 2006.

[15] S. Liscano, L. Gil y R. Subero. "Evaluación de la resistencia al desgaste abrasivo y deslizante de recubrimientos de alúmina-13\% titania termorrociados sellados". UCT, Vol. $10 \mathrm{~N}^{\circ} 39$, pp. 178-180. Julio 2006. 
[16] K. Holmberg, H. Ronkainen, A. Laukkanen and K. Wallin. "Friction and wear of coated surfaces-scales, modeling and simulation of tribomechanisms". Surface \& Coating Technology. Vol. 202, Issue 4-7, pp. 10341049. December, 2007.

[17] D.E. Salazar and C.M. Rocco. "Solving advanced multi-objective robust designs by means of multiple objective evolutionary algorithms (MOEA): A reliability application". Reliability Engineering \& System Safety. Vol. 92, Issue 6, pp. 697-706. June, 2007.

[18] D.C. Montgomery. "Diseño y análisis de experimentos". Limusa Wiley. México. 2003.

[19] W. Li, S.J. Hu and S.W. Cheng. "Robust Design and Analysis for Manufacturing Processes with Parameter Interdependency". Journal of Manufacturing Systems. Vol. 21, Issue 2, pp. 93-100. 2002.

[20] D.H. Wu and M.S. Chang. "Use of Taguchi method to develop a robust design for the magnesium alloy die casting process".
Materials Science and Engineering A. Vol. 379, Issue 1-2, pp. 366-371. August, 2004.

[21] D.C. Chen and C.F. Chen. "Use of Taguchi method to develop a robust design for the shape rolling of porous sectioned sheet". Journal of Materials Processing Technology. Vol. 177, Issue 1-3, pp. 104-108. July 3, 2006.

[22] D.C. Chen and C.F. Chen. "Use of Taguchi method to study a robust design for the sectioned beams curvature during rolling". Journal of Materials Processing Technology. Vol. 190, Issue 1-3, pp. 130-137. July 23, 2007.

[23] H. Gutiérrez y S.R. de la Vara. "Análisis y diseño de experimentos". McGraw Hill. México. 2004.

[24] C.U. Yu, C.-C. Hu, A. Bai and Y.F. Yang. "Pore-size dependence of AAO films on surface roughness of Al1050 sheets controlled by electropolishing coupled with fractional factorial design". Surface and Coating Technology. Vol. 201, Issue 16-17, pp. 72597265. May 21, 2007. 